\title{
Raman Nonlinearity Contribution to Spatio-Temporal Pulse Dynamics Under Filamentation in Yag Crystal
}

\section{Kirill V. Lvov'1,2, Sergey Yu. Stremoukhov'1,2, and Fedor V.Potemkin}

${ }^{1}$ Faculty of Physics, M.V. Lomonosov Moscow State University, Moscow, Russia

${ }^{2}$ National Research Centre "Kurchatov Institute", Moscow, Russia

\section{Abstract}

We report on numerical study of the nonlinear pulse propagation under filamentation of femtosecond near-infrared radiation under normal dispersion regime in YAG crystal. Influence of the Raman and Kerr tandem action on a spatial and temporal dynamics of the laser pulse were discussed. We have shown that Raman nonlinearity provides an asymmetry of temporal pulse profile: the leading edge becomes more bright and

Corresponding Author: Kirill V. Lvov

Ivov.kv14@physics.msu.ru

Received: 28 January 2018

Accepted: 15 March 2018

Published: 25 April 2018

Publishing services provided by Knowledge

(c) Kirill V. Lvov et al. This article is distributed under the terms of the Creative Commons

Attribution License, which permits unrestricted use and redistribution provided that the original author and source are credited.

Selection and Peer-review under the responsibility of the PhIO Conference Committee.

\section{G OPEN ACCESS}

propagates at larger distances in comparison with trailing edge.

Keywords: filamentation, supercontinuum generation, Raman nonlinearity.

\section{Introduction}

An extensive study of femtosecond laser filamentation is up to date stimulated by the numerous applications arising from the highly nonlinear nature of this process. Combined action of the Kerr nonlinearity and plasma defocusing in air allows laser pulse propagation up to kilometers distances giving an opportunity for measuring atmospheric trace species [1]. Extended spatial confinement of femtosecond laser beam and high peak intensity inside the filament can be beneficial for the development of nitrogen-based remote lasers [2]. A complex interplay between self-action processes (self-phase modulation, self-steepening, etc.) and plasma generation results in multi-octave spectral broadening [3]. Laser filamentation in anomalous dispersive media is considered as a powerful way to produce few-cycle pulses in near and mid-IR [4], whereas two-color filamentation is used for generation of $\mathrm{THz}$ radiation [5].

Seeding optical parametric devices with supercontinuum (SC) is widely used technique since its greatly reduce amplification threshold and improve energy stability. For this purpose white-light generation stage based on pump pulse spectral broadening in transparent dielectrics is usually applied. YAG crystal is the material of choice for SC 
generation because its high nonlinear refractive index (compared to sapphire, quartz, etc.) and high optical damage threshold. The SC generation with femtosecond near-IR laser pulses in this crystal was widely studied experimentally and theoretically [6]. However, in several early published papers [7] appearance of the distinct peaks in SC spectrum was observed. The origin of these peaks stays unclear and requires detailed numerical study.

In this paper, we analyze numerical model, which describes pulse propagation under filamentation of femtosecond laser pulses centered at $1240 \mathrm{~nm}$ (that corresponds to

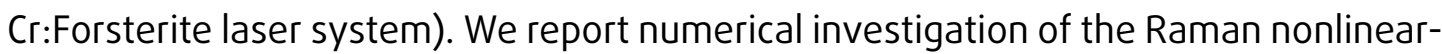
ity influence on spatio-temporal pulse dynamics. Our results show that this nonlinear term leads to asymmetrical pulse profile: energy shifting toward the leading edge.

\section{Numerical simulation}

Theoretical model, which describes SC generation process in YAG crystal, is relied on paraxial approximation, according to which wave vector of incident wave is directed along with pulse propagation axis, and also on slowly varying envelope approximation (SVEA), according to which magnitude of electrical field $A$ varies weakly at the distance being propagated by wave during one cycle: $\left|\frac{\partial A}{\partial z}\right|<<\frac{\omega}{v_{g}}|A|$. Equation, which describes spatial and temporal changings in magnitude of electrical field, is a consequence of Maxwell's equations and reads as:

$$
\frac{\partial A}{\partial z}=\frac{i}{z k_{0}} \Delta_{\perp} A-\frac{k^{\prime}{ }_{0}}{2} \frac{\partial^{2} A}{\partial t^{2}}+\left(N_{\text {kerr }}+N_{\text {MPA }}+N_{\mathrm{pl}}\right) A,
$$

where $\lambda=1240 \mathrm{~nm}$ is the wavelength, $n_{0}=1,8$ is the linear refractive index, $k_{0}=\frac{\omega n_{0}}{c}$ is wave number, $k{ }_{0}{ }_{0}=428 \mathrm{fs}^{2} / \mathrm{cm}$ is the group velocity dispersion. In (1) under $t$ we mean retarded time for the pulse traveling at the group velocity $v_{g}: t \rightarrow t-\frac{z}{v_{g}}$.

The first term in the right part of (1) describes diffraction in medium, the second one - the second order of dispersion, the third one - the Kerr and Raman nonlinearities (2), the forth one - multiphoton absorption (MPA) (3), the fifth one - nonlinearity induced by plasma generation (4).

$$
\begin{gathered}
N_{\text {kerr }}=\frac{3 \pi}{2} \frac{n_{2}}{\lambda} i\left((1-\theta)|A|^{2}+\theta \int_{-\infty}^{t} \frac{r^{2}+\omega_{R}^{2}}{\omega_{R}} e^{-\Gamma i(t-\tau)} \sin \omega_{R}(t-\tau)|A(\tau)|^{2} d \tau\right) \\
N_{\mathrm{MPA}}=-\frac{\beta_{k}}{2}|A|^{2 K-2}\left(1-\frac{\rho}{\rho_{\text {at }}}\right), \\
N_{\mathrm{pl}}=-\frac{\sigma}{2}\left(1+i \omega_{0} \tau_{c}\right) \rho,
\end{gathered}
$$


where $n_{2}=6,9 \cdot 10^{-16} \mathrm{~cm}^{2} / \mathrm{W}$ is the nonlinear refraction index, $\omega_{R}=1,09 \mathrm{fs}^{-1}$ is the Raman frequency, $\beta_{k}=4 \cdot 10^{-74} \mathrm{~W}^{-6} \mathrm{~cm}^{11}$ is the ionization coefficient, $K=7$ is the order of multiphoton ionization [8], $\rho_{\text {at }}=2,1 \cdot 10^{22} \mathrm{~cm}^{-3}$ is the neutral atom density, $\sigma=5,6 \cdot 10^{-18} \mathrm{~cm}^{2}$ is the cross section for inverse bremsstrahlung, $\tau_{c}=1,7 \mathrm{fs}$ is the collision time. Taking into account only particular number of effects governs spatial and temporal dynamics of envelope (for example, the second order of dispersion instead of the whole dispersion in frequency domain) let us separate and understand the contribution of each of these terms.

Kerr and Raman contribution into nonlinearity caused by two factors: "instantaneous" electron response (the Kerr nonlinearity) and slow exiting vibration molecules levels with decrement $\Gamma=96 \mathrm{fs}$ (the Raman nonlinearity). Parameter $\theta$ characterizes the relative influence of Kerr and Raman contribution on whole nonlinearity.

The rate equation for electron density includes multiphoton ionization, avalanche ionization and electron recombination and can be expressed as:

$$
\frac{\partial \rho}{\partial t}=\left(\frac{\beta_{k}}{K \hbar \omega_{0}}|A|^{2 K}+\frac{\sigma}{U_{\text {ion }}} \rho|A|^{2}\right)\left(1-\frac{\rho}{\rho_{\text {at }}}\right)-\frac{\rho}{\tau_{\Gamma}},
$$

where $U_{\text {ion }}=6,5 \mathrm{eV}$ is the YAG bandgap, $\tau_{\Gamma}=1 \mathrm{ps}$ is the recombination time [9].

For numerical calculations we use Gaussian in space (FWHM equals $w_{o}=0,05 \mathrm{~cm}$ ) and time (FWHM equals $t_{p}=200 \mathrm{fs}$ ) initial pulse focused into the crystal $5 \mathrm{~mm}$ deep into the sample surface by the lens with focal distance $200 \mathrm{~mm}$. Critical radiation power needed to self-focusing reads as $P_{c r}=0,9 \mathrm{MW}$ and critical intensity reads as $I_{c r}=0,17$ $\mathrm{GW} / \mathrm{cm}^{2}$.

\section{Results and discussion}

Firstly, we investigated the influence of incident pulse energy on propagation process. For this purpose, we calculated the distribution of radiation intensity for the different laser pulse energy at the propagation axis. When pulse power exceeds critical power $P_{c r}$, nonlinear focus at propagation axis due to the Kerr effect is formed (Fig.1a), electron density rapidly increases (Fig.1d) and, as a result, energy emerges out of the pulse center. During further propagation, due to the Kerr effect a new nonlinear focus is formed, electron density again increases, and refocusing cycles repeat as pulse propagates along the z-axis. The pulse energy increases, a number of nonlinear foci increases and its shift towards the front side of the crystal, since greater energy is pumped into the system, the intensity of radiation is higher and the Kerr nonlinearity acts more intensively. 

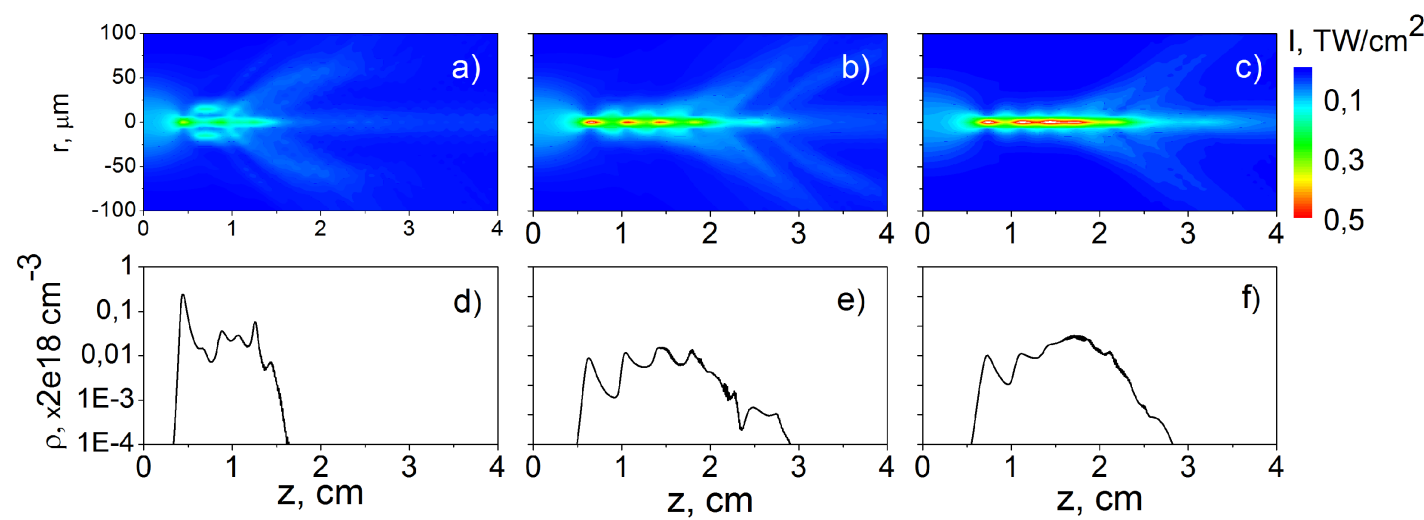

Figure 1: At the top: distribution of radiation intensity averaged over time for pulse power 15 Pcr. The Raman nonlinearity contribution at the level a) $\theta=0, b) \theta=0.2, c) \theta=0.4$. At the bottom: electron density at the axis for pulse power 15 Pcr. The Raman nonlinearity contribution at the level $a) \theta=0, b) \theta=0.2, c) \theta$ $=0.4$.

Comparison of intensity distribution for Raman nonlinearity level $\theta$ from 0 to 0.4 is depicted in Fig.1. Note, that these distributions are averaged over time. In the absence of Raman nonlinearity there is only one well-marked focusing point and in addition one or two faint focuses. Such pattern is observed due to plasma generation and, as a result, strong defocusing of initial pulse radiation. When the Raman nonlinearity acts, one may see a sequence of nonlinear foci, for instance, 4 foci at Fig.1b. Electron density profiles at the bottom of Fig.1 follows up the intensity distribution. We conclude that the Raman nonlinearity leads to decreasing of ionization degree and, as a result, to less defocusing and propagation at longer distances, which may affect on the conversion efficiency of the generated along with the laser propagation radiation.

In Fig.2 there are depicted radiation intensity distributions at the axis of propagation in the dependence on z-coordinate and retarded time $t$ for the various Raman nonlinearity levels. Since pulse propagates through media with normal group velocity dispersion, initially Gauss-shape pulse splits into two subpulses, the leading one propagates with higher and the trailing one - with lower group velocity in comparison with that is for central wavelength. In the absence of the Raman nonlinearity there is deformation of the trailing edge (Fig.2a): it becomes steeper, that is caused by plasma generation. Moreover, when pulse energy is high enough, the radiation intensity of the trailing subpulse becomes so high, that the secondary temporal splitting occurs. If the Raman nonlinearity is taken into account, there is asymmetry in time-domain: the leading subpulse has higher intensity and propagates at longer distance in comparison with the trailing subpulse. In Fig.2b and $2 \mathrm{c}$ the first focusing point even has not well-marked trailing edge. It is important to notice qualitative difference between effects induced by plasma generation and the Raman nonlinearity. The first one also 
contributes to asymmetrical temporal pulse profile, but energy at the trailing edge is absorbed, whereas the second one leads to energy shifting toward the leading edge. Such pattern can be seen by comparison of intensity distribution in Fig.2a and 2c. Another feature consists in pulse center lad in time domain that is clearly seen in Fig.2c. Notice that as follow from Fig.1 when $\theta=0.2,0.4$, electron density is less by 1-2 orders of magnitude than that for $\theta=0$, i.e. there is only the Raman nonlinearity influence on intensity distribution in Fig.2b and $2 c$.
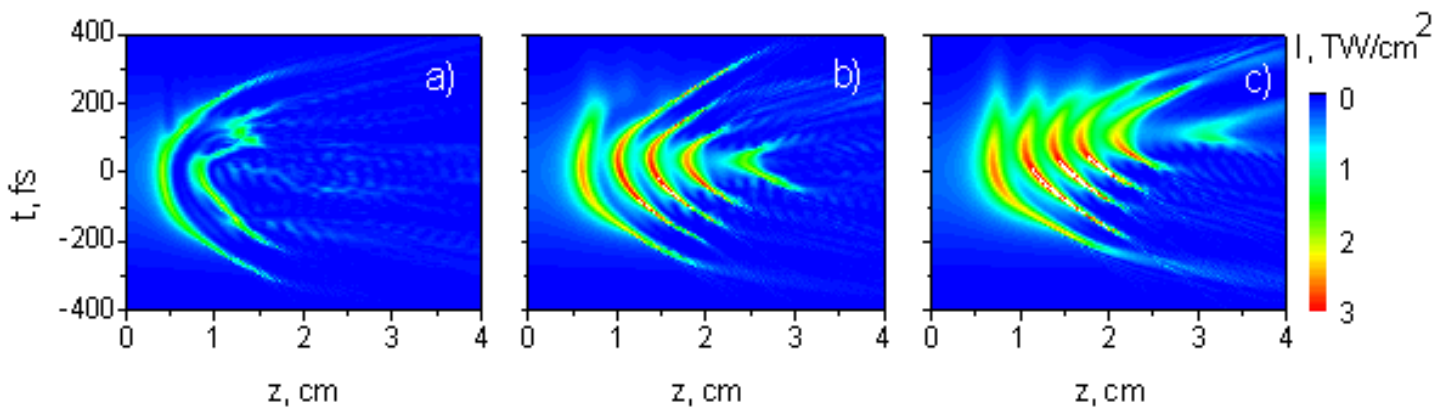

Figure 2: Distribution of radiation intensity at the axis for pulse power 15 Pcr. Raman nonlinearity contribution at the level a) $\theta=0, b) \theta=0.2, c) \theta=0.4$.

In Fig.3 spatio-temporal pulse profiles (STPP) at the distances $0.5,1 \mathrm{n} 1.5 \mathrm{~cm}$ from the front side of crystal are depicted. Fig 3 (a-c) show the STPP in the absence of Raman nonlinearity. In Fig.3a (corresponds to the beginning of the first pulse splitting event) after passing the subpulse radiation the radial distribution of the laser pulse intensity becomes more complicated, due to self-focusing of the pulse the central time slices suffers from the decreasing of the velocity in comparison with the peripheral ones. During further pulse propagation, new splitting events occur and additional subpulses are formed. Fig.3 (d-f) show the STPP correspond to $\theta=0.4$, distances from the front side of crystal are also $0.5,1$ и $1.5 \mathrm{~cm}$. For the case of $z=0.5 \mathrm{~cm}$ (Fig.3d), pulse intensity increased but there is no pulse splitting. At $z=1 \mathrm{~cm}$ (Fig. $3 \mathrm{e}$ ) pulse splitting has occurred and the central time slices are defocused together with the pulse tail. At $z=$ $1.5 \mathrm{~cm}$ (Fig. $3 \mathrm{f}$ ) pulse profile considerably becomes more complicated due to additional nonlinear focus and splitting events. But steep leading edge and sloping trailing edge are well noticeable that proves the earlier conclusion about the Raman nonlinearity influence. 


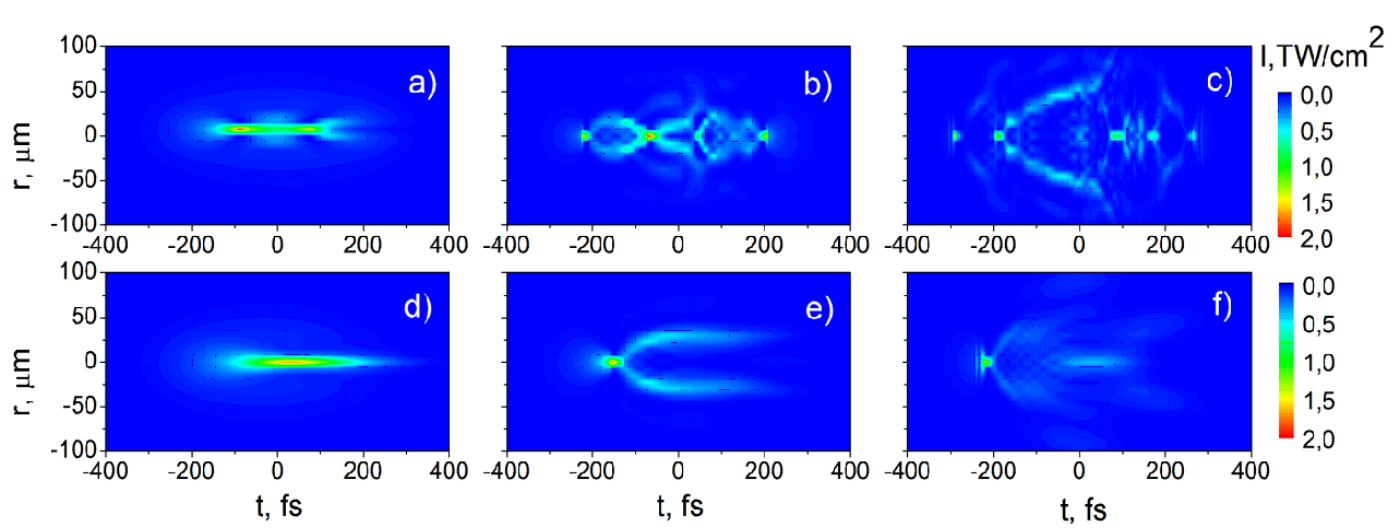

Figure 3: At the top: distribution of radiation intensity for pulse power $15 \mathrm{Pcr}$ and $\theta=0$ at $\mathrm{a}) \mathrm{z}=0.5 \mathrm{~cm}, \mathrm{~b}) \mathrm{z}$ $=1 \mathrm{~cm}, \mathrm{c}) \mathrm{z}=1.5 \mathrm{~cm}$. At the bottom: distribution of radiation intensity for pulse power $15 \mathrm{Pcr}$ and $\theta=0.4$ at $d) z=0.5 \mathrm{~cm}, e) z=1 \mathrm{~cm}, \mathrm{f}) \mathrm{z}=1.5 \mathrm{~cm}$.

\section{Conclusion}

In conclusion, we have studied nonlinear propagation of femtosecond near-infrared laser pulses in YAG crystal and have revealed the Raman nonlinearity influence on spatio-temporal pulse dynamics. In normal dispersive regime splitting of initial laser pulse into two subpulses occurs. We have shown that the Raman nonlinearity leads to asymmetrical temporal pulse profile: energy shifting toward the leading edge and emerging out of the pulse center. As a result, the number of nonlinear foci along the $z$-axis increases. Analyzing the dynamics of the radial intensity distributions we have revealed the complicated nature over the propagation coordinate. In the origin of such behavior the prevailing factor is the plasma defocusing of the time slices of the laser pulse. The tandem action of the plasma and the Raman nonlinearities can bring some benefits to the radial pulse profile control.

This work was supported by the Russian Scientific Fund (Grant no. 17-72-20130).

\section{References}

[1] A. Couairon, A. Mysyrowicz. Physics Reports. Vol. 441, 47 - 189 (2007).

[2] J. Yao, B. Zeng et al. Phys. Rev. A. Vol. 84 (2011).

[3] M. Bradler, P. Baum, E. Riedle. Appl Phys B, Vol. 97: 561-574 (2009).

[4] S.V. Chekalin et al. Quantum Electronics Vol. 47, No. 3, $252-258$ (2017).

[5] S.I. Mitryukovskiy et al. Appl. Phys. Let. Vol. 102 (2013).

[6] V. Jukna et al. Appl. Phys. B. Vol. 116, No. 2, 477-483 (2014). 
[7] N. Garejev, G. Tamosauskas. Journal of the Optical Society of America B, Vol. 34, No. 1 (2017).

[8] L.V. Keldysh. JETP. Vol. 20, No. 5 (1965).

[9] J. Galinis, G. Tamosauskas et al. Phys. Rev. A. Vol. 92 (2015).

[10] N. Garejev et al. Optics Express. Vol. 24, No. 15 (2016). 